\title{
Análisis de la calidad de la información proporcionada a los pacientes por parte de unidades clínicas especializadas ambulatorias mediante análisis por modelos multinivel
}

\section{Analysis of the quality of the information supplied to the patients by specialised outpatient clinical units by means of multilevel model analysis}

\section{Rodrigo ${ }^{1}$, J.J. Viñes, F. Guillén-Grima ${ }^{2}$}

\section{RESUMEN}

Fundamento. La influencia de la información recibida por los pacientes en su satisfacción es de gran importancia ya que, entre otros aspectos, puede mejorar la adherencia del paciente al tratamiento y contribuir a mejorar su estado de salud.

El objetivo del estudio es conocer, a partir de los cuestionarios de satisfacción, qué porcentaje de la variabilidad de la percepción del paciente sobre la información recibida acerca del régimen de vida y los medicamentos se atribuye a los siguientes niveles: paciente, unidad clínica y especialidad; así como identificar las variables que han influido en los resultados.

Material y métodos. Se realizaron entrevistas telefónicas a 6.922 pacientes atendidos en consultas externas en el Servicio Navarro de Salud en el año 2005 (tasa de respuesta $92,4 \%$ ). Se ha evaluado si los pacientes habían recibido prescripción farmacológica e información sobre el régimen de vida y, en caso afirmativo, su valoración de la calidad de la mencionada información. Se han analizado 94 unidades clínicas en régimen ambulatorio y 34 especialidades médicas. Se ha realizado un análisis estadístico jerárquico con 3 niveles.

Resultados. En los ítems estudiados, el porcentaje de la variabilidad explicada por la unidad clínica y la especialidad médica osciló entre 0 y el $12,6 \%$ y el explicado por el paciente, entre el $87,4 \%$ y el $99,3 \%$.

Conclusiones. Se ha detectado variabilidad en el comportamiento de las distintas unidades clínicas en relación con la prescripción farmacológica y con la información proporcionada sobre el régimen de vida. No obstante, la mayor parte de la variabilidad se ha localizado en el nivel "paciente".

Palabras clave. Satisfacción del paciente. Análisis multinivel. Prescripción farmacológica. Régimen de vida. Unidades clínicas.

\begin{abstract}
Background. The influence of the information received by patients on their satisfaction is of great importance since, amongst other aspects, it can improve patients' adherence to treatment and contribute to improving their state of health.

The study aimed to determine, on the basis of satisfaction questionnaires, the percentage of variability in patients' perception of the information received concerning way of life and medicines, attributed to the following levels: patient, clinical unit and specialisation. It also aimed to identify the variables that influence the results.
\end{abstract}

Material and Methods. Telephone interviews were conducted with 6,922 patients treated in outpatient units in the Navarre Health Service in the year 2005 (rate of reply, 92.4\%). An evaluation was made of whether the patients had received medical prescription and information on way of life and, where affirmative, their evaluation of the quality of this information. Ninety-four outpatient clinics and 34 medical specialisations were analysed. A hierarchical statistical analysis at three levels was made.

Results. In the items studied, the variability explained by clinical unit and medical specialisation oscillated between 0 and $12.6 \%$, and explained by the patient between 87.4 and $99.3 \%$.

Conclusions. Variability was detected in the behaviour of the different clinical units in relation to medical prescription and information provided on way of life. However, the greater part of the variability was localised at the "patient" level.

Key words. Patient satisfaction. Multilevel analysis. Medical prescription. Way of life. Clinical units.

\section{An. Sist. Sanit. Navar. 2009; 32 (2): 183-197}

1. Servicio de Medicina Preventiva y Gestión de Calidad. Hospital de Navarra. Pamplona.

2. Departamento de Ciencias de la Salud. Universidad Pública de Navarra. Pamplona.

Recepción: 5 de febrero de 2009

Aceptación provisional: 26 de marzo de 2009

Aceptación definitiva: 29 de marzo de 2009

\author{
Correspondencia \\ $M^{a}$ Isabel Rodrigo Rincón \\ Servicio de Medicina Preventiva y Gestión de la \\ Calidad \\ Hospital de Navarra \\ Irunlarrea, 3 \\ 31008 Pamplona \\ Tfno. 848422499 \\ E-mail: mi.rodrigo.rincon@cfnavarra.es
}




\section{INTRODUCCIÓN}

Una de las variables que con mayor frecuencia se estudia en las encuestas de satisfacción es la información que proporcionan los profesionales sanitarios a los pacientes $^{1-3}$.

Esta información es de una gran importancia, por una parte, porque puede inducir cambios en el comportamiento de los pacientes hacia hábitos más saludables ${ }^{4,5}$, y por otra, porque es uno de los elementos que puede influir en la satisfacción de los pacientes. De hecho, en una revisión realizada por Williams, Weinman y Dale ${ }^{6}$ sobre la relación entre la comunicación entre paciente y médico y la satisfacción del paciente, encontraron que una mayor información proporcionada por los facultativos se asociaba con una mayor satisfacción de los pacientes. Es más, en el ámbito de la atención especializada, la información proporcionada por el médico era el elemento que mejor predecía la satisfacción de los pacientes.

El consejo y la información sobre hábitos de vida (cesación tabáquica, dieta, ejercicio, etc.), al sugerir cambios en sus conductas, podrían "molestar" a los pacientes. Sin embargo, se ha comprobado que esta información puede mejorar su satisfacción ${ }^{7}$.

En cuanto a los medicamentos prescritos, la mayoría de los pacientes desean que los facultativos les proporcionen información completa sobre los mismos, incluyendo los efectos adversos que se puedan derivar de su administración ${ }^{8}$.

Respecto a la validez de la opinión de los pacientes en relación a la información recibida, se ha encontrado una correlación positiva entre la valoración que realizan los pacientes y la información realmente proporcionada registrada mediante la grabación de los encuentros médicos ${ }^{1}$.

Una mayor satisfacción de los pacientes mejora el cumplimiento de la agenda de citación, el uso de los medicamentos prescritos y la intención de cumplir con las recomendaciones proporcionadas ${ }^{9}$, lo que contribuiría a mejorar el estado de salud de los pacientes ${ }^{10}$.
Aunque hay numerosos trabajos que analizan la percepción de los pacientes sobre la información proporcionada ${ }^{3-4}$, se ha explorado poco qué peso juegan las especialidades médicas y las unidades clínicas en la variabilidad de las puntuaciones obtenidas y todavía es poco frecuente el empleo del análisis multinivel para identificar en las encuestas de calidad percibida qué unidades organizativas se sitúan por encima o por debajo de la media.

El objetivo del estudio es conocer, a partir de los cuestionarios de satisfacción, qué porcentaje de la variabilidad de la percepción del paciente sobre la información recibida acerca del régimen de vida y los medicamentos se atribuye a cada uno de los siguientes niveles: paciente, unidad clínica y especialidad; así como identificar las variables que han influido en la obtención de los resultados. Para ello se ha utilizado una estructura multinivel en el que el paciente (nivel 1) se anida en unidades clínicas (nivel 2) y éstas en especialidades médicas (nivel 3).

\section{MATERIAL Y MÉTODOS}

Los datos utilizados proceden de un estudio transversal en el que a través de una entrevista telefónica con un cuestionario estructurado se preguntó a 6.922 pacientes, atendidos en consultas ambulatorias, su percepción sobre diferentes aspectos de la atención recibida. El cuestionario consta de 25 preguntas, excluyendo las referentes a datos personales y de control, según un modelo predefinido de preguntas cerradas de opción múltiple al que se añadió una pregunta abierta para recoger las sugerencias de los usuarios. Las preguntas incluidas en el cuestionario hacen referencia a aspectos en los que los profesionales que trabajan en las mencionadas unidades clínicas tienen algún grado de responsabilidad. No se preguntaron otros aspectos como la limpieza, hostelería, parking, etc. ya que son aspectos comunes para el centro en su conjunto y, por lo tanto, no son específicos de las unidades clínicas. 
El trabajo de campo fue realizado por una empresa especializada. Para la realización de las entrevistas se contó con la participación de 10 encuestadores que cubrieron un horario, a doble turno, de 9 de la mañana a 9 de la noche, que permitió intentar establecer contacto hasta en diez ocasiones con todos los pacientes susceptibles de ser entrevistados.

Las entrevistas se realizaron un mes después del contacto sanitario. Previamente a la entrevista, el paciente recibió una carta en su domicilio informándole de los objetivos de la encuesta.

La tasa de respuesta al cuestionario fue del $92,4 \%$. En cuanto a la fiabilidad del cuestionario, los coeficientes alfa de Cronbach y el coeficiente alfa con los ítems tipificados alcanzaron un valor de 0,96 . Este mismo coeficiente alfa se obtuvo para las preguntas "calidad de información de medicamentos" y "calidad de información régimen de vida" tras la exclusión de cada ítem. La correlación múltiple al cuadrado obtuvo un valor de 0,65 para la variable "calidad de información de medicamentos" y de 0,64 para "calidad de la información sobre el régimen de vida".

La validez de criterio concurrente respecto al criterio de referencia "fidelidad"11-13 (preguntaba si el paciente volvería al mismo servicio en el caso de que en un futuro necesitase ser atendido por un problema similar y pudiera elegir) obtuvo un coeficiente de correlación Rho de Spearman de 0,52 para el ítem "calidad de la información sobre el régimen de vida" y de 0,56 para el ítem sobre "calidad de la información sobre medicamentos".

Entre octubre y diciembre del año 2005 fueron entrevistados 6.922 pacientes atendidos en 94 unidades clínicas médicas (37 especialidades médicas) del Servicio Navarro de Salud-Osasunbidea (SNS-O). La unidad muestral fue la unidad clínica extrayéndose de cada una de ellas una muestra de pacientes mediante un muestreo aleatorio simple sin reposición. Para cada unidad clínica se seleccionaron un mínimo de 50 y un máximo de 100 pacientes.
Se definió como unidad clínica aquella unidad de atención ambulatoria especializada compuesta por sus recursos materiales y humanos que atiende a los pacientes de una especialidad en un centro sanitario (ejemplo, unidad de cardiología que orgánicamente pertenece al centro X). Se definió como especialidad a la agrupación de unidades clínicas que tratan a los pacientes de una misma especialidad en el conjunto del SNS-O (ejemplo: cardiología).

Para este estudio se han analizado las preguntas que hacen referencia a la información recibida sobre medicamentos y sobre el régimen de vida. Al paciente se le preguntó si le habían recetado algún medicamento en la consulta o si le habían proporcionado información sobre el régimen de vida. En el caso de que la respuesta fuera afirmativa, se pedía al paciente que valorara en una escala de 0 al 10 (0 peor puntuación posible, 10 mejor puntuación posible) la calidad de la información recibida.

Se estudiaron tanto variables individuales de los pacientes: edad, género y tipo de consulta, como específicas de cada unidad clínica: tipo de unidad, tamaño y tipo de centro al que pertenecen.

Para el análisis de la percepción de la calidad de la información sobre el régimen de vida y sobre medicamentos solamente se tuvieron en cuenta los sujetos que respondieron haber recibido algún tipo de información o haber recibido prescripción farmacológica.

\section{Análisis estadístico}

Se realizó un análisis multinivel con las aplicaciones informáticas STATA 10 y MLWIN 2.10. Para el resto de análisis se utilizó SPSS 15.0.

El análisis multinivel se estructuró en 3 niveles (Fig. 1). El nivel 1 lo constituyen los pacientes, el nivel 2 las unidades clínicas y el nivel 3 las especialidades médicas. Los pacientes se anidan en las unidades clínicas y las unidades clínicas se anidan en especialidades médicas. 


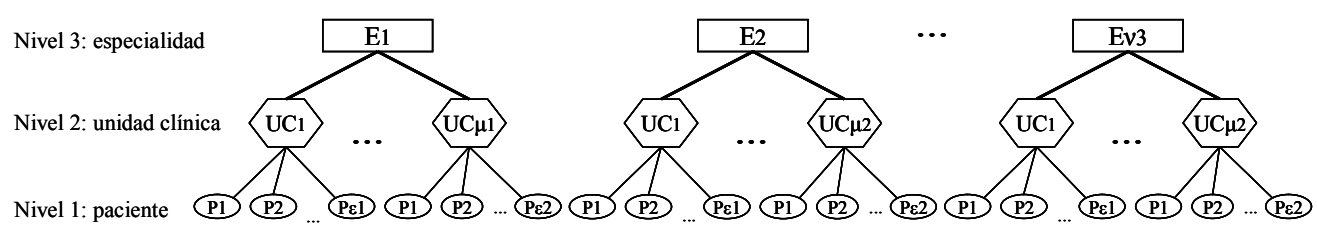

Figura 1. Representación del análisis jerárquico o multinivel.

Se han realizado diversos análisis multinivel $^{14}$. En primer lugar, se ha utilizado un modelo nulo sin ningún tipo de variables predictoras. Para las variables dependientes cuantitativas el modelo nulo se define de la siguiente manera:

$$
\mathrm{Y}_{\mathrm{ijk}}=\beta_{000}+\eta_{\mathrm{k}}+\mu_{\mathrm{jk}}+\varepsilon_{\mathrm{ijk}}
$$

- $\mathrm{Y}_{\mathrm{ijk}}$ representa el valor de la variable dependiente, por ejemplo, información sobre el régimen de vida, para el paciente $i$, en la unidad clínica $j$ de la especialidad médica $k$.

- $\beta_{000}$ representa la media general y los términos aleatorios $\eta_{\mathrm{k},} \mu_{\mathrm{jk}} \mathrm{y} \varepsilon_{\mathrm{ijk}}$ representan los residuales de cada nivel: especialidad médica, unidad clínica y paciente respectivamente. Se asume que los términos aleatorios tienen una media de 0 y una varianza de $\tau_{\mathrm{h}}, \tau_{\mu} \mathrm{y}_{\varepsilon}^{2}$ para los niveles 3,2 y 1 respectivamente.

El modelo se va construyendo por etapas, introduciendo las variables de los diferentes niveles de forma que el modelo final vendría representado por la siguiente ecuación:

$$
\mathrm{Y}_{\mathrm{ijk}}=\beta_{000}+\beta \mathrm{X}_{\mathrm{ijk}}+\alpha \mathrm{W}_{\mathrm{jk}}+\gamma \mathrm{Z}_{\mathrm{k}}+\eta_{\mathrm{k}}+\mu_{\mathrm{jk}}+\varepsilon_{\mathrm{ijk}}
$$

Siendo $\beta$ el coeficiente de las variables del nivel 1 (paciente) y $\alpha$ y $\gamma$ los coeficientes para las variables a nivel de unidad clínica (nivel 2) y especialidad médica (nivel 3) respectivamente.

Para las variables dependiente dicotómicas, el modelo matemático se representa con la siguiente ecuación:

$$
\operatorname{logit}\left[\varpi_{i j \mathrm{k}}\right]=\beta_{000}+\beta \mathrm{X}_{\mathrm{ijk}}+\alpha \mathrm{W}_{\mathrm{jk}}+\gamma \mathrm{Z}_{\mathrm{k}}+\eta_{\mathrm{k}}+\mu_{\mathrm{jk}}+\varepsilon_{\mathrm{ijk}}
$$

Se ha estudiado la parte fija del modelo mediante el análisis de las variables explicativas a través de la razón t definida como el coeficiente estimado dividido por su error estándar. En las tablas solamente se reflejan aquellas variables cuyos coeficientes son estadísticamente significativos.

La parte aleatoria del modelo se ha estudiado mediante el análisis de la variabilidad atribuible al paciente, a la unidad clínica, y a la especialidad. El coeficiente de partición de la varianza (CPV) mide la proporción de la varianza que es debida a la diferencia entre los grupos, es decir, la cantidad de dependencia o la proporción de la varianza que explica la estructura grupal.

Se ha calculado el CPV según el método de Siddiqui, Hedeker, Flay y $\mathrm{Hu}^{15}$ de la siguiente forma:

- $\rho$ primer nivel $=\sigma_{\varepsilon}^{2} /\left(\tau_{h}+\tau_{\mu}+\sigma_{\varepsilon}^{2}\right)$

- $\rho$ segundo nivel $=\left(\tau_{\mathrm{h}}+\tau_{\mu}\right) /\left(\tau_{\mathrm{h}}+\tau_{\mu}+\sigma_{\varepsilon}^{2}\right)$

- $\rho$ tercer nivel $=\tau_{h} /\left(\tau_{h}+\tau_{\mu}+\sigma_{\varepsilon}^{2}\right)$

Los valores del CPV oscilan entre 0 (no hay diferencias entre los grupos) y 1 (no hay diferencias entre los individuos de cada grupo).

En la distribución logística la varianza para el primer nivel se ha calculado ${ }^{16}$ con la siguiente fórmula: $\varpi^{2} / 3=3,29$.

En el análisis multinivel la estimación de los parámetros (coeficientes de regresión y componentes de la varianza) se ha realizado a través del método de máxima verosimilitud $^{15-18}$. El procedimiento de máxima verosimilitud genera un estadístico denominado desvianza que indica la bondad del ajuste del modelo. El valor de la desvianza es menos dos veces el logaritmo de la verosimilitud. Los modelos con desvianza más pequeña consiguen un mejor ajuste que los modelos con mayor valor de desvianza. La desvianza sigue una distribución Ji cuadrado en la que los grados de libertad se corresponden con la diferencia entre el número de parámetros de los dos modelos comparados ${ }^{19}$. 
Para cada variable dependiente se han explorado 4 modelos. No obstante, en los resultados solamente se presentan el modelo vacío, sin variables independientes, y el modelo completo, con todas las variables predictoras disponibles. Los modelos intermedios no han conseguido mejorar el valor de la desvianza con respecto al modelo completo.

En cada modelo se ha calculado: el valor de la desvianza, el valor de la varianza y su error estándar, el porcentaje de la variabilidad explicada por la unidad clínica y por la especialidad, así como la variabilidad de las unidades clínicas atribuible a la especialidad.

Para cada variable dependiente se ha calculado, utilizando el análisis multinivel, el valor del residual del intercepto de las distintas unidades clínicas mediante la diferencia entre la media del intercepto de cada unidad clínica y la media general. También se ha calculado el intervalo de confianza del residual al $95 \%$. Se ha considerado que una unidad clínica obtiene valores diferentes a la media general cuando en el intervalo de confianza de su residual no incluye el valor 0 .

Para todas las pruebas estadísticas se ha fijado el valor crítico o nivel de significación en el $5 \%$.

\section{RESULTADOS}

En la tabla 1 se presentan las principales características de los individuos encuestados.

Tabla 1. Número y porcentaje de pacientes entrevistados en función de las variables predictoras.

\begin{tabular}{|c|c|c|c|c|c|c|}
\hline & Número & $\%$ & $\begin{array}{l}\text { \% de pacientes } \\
\text { con prescripción } \\
\text { farmacológica }\end{array}$ & $\begin{array}{l}\text { Puntuaciones } \\
\text { medias de } \\
\text { información sobre } \\
\text { medicamentos } \\
\text { (Escala 0-10) }\end{array}$ & $\begin{array}{l}\text { \% de pacientes } \\
\text { con información } \\
\text { sobre régimen } \\
\text { de vida }\end{array}$ & $\begin{array}{l}\text { Puntuaciones } \\
\text { medias de } \\
\text { información sobre } \\
\text { régimen de vida } \\
\text { (Escala 0-10) }\end{array}$ \\
\hline \multicolumn{7}{|l|}{ Género } \\
\hline Hombre & 3.016 & 43,6 & 50,8 & 8,7 & 26,4 & 8,6 \\
\hline Mujer & 3.906 & 56,4 & 47,2 & 8,7 & 22,5 & 8,4 \\
\hline \multicolumn{7}{|l|}{ Edad } \\
\hline Menos de 16 años & 255 & 3,7 & 34,8 & 8,8 & 16,1 & 8,1 \\
\hline Entre 16 y 65 años & 4.243 & 61,3 & 44,4 & 8,6 & 23,2 & 8,5 \\
\hline Más de 65 años & 2.424 & 35,0 & 57,7 & 8,9 & 24,1 & 8,6 \\
\hline \multicolumn{7}{|l|}{ Tipo de consulta } \\
\hline Primera consulta & 2.475 & 35,8 & 39,9 & 8,7 & 19,2 & 8,5 \\
\hline Consulta sucesiva & 4.432 & 64,2 & 53,7 & 8,7 & 26,9 & 8,5 \\
\hline \multicolumn{7}{|l|}{ Tipo de servicio } \\
\hline Médico & 4.933 & 71,3 & 50,5 & 8,7 & 26,1 & 8,5 \\
\hline Quirúrgico & 1.989 & 28,7 & 44,4 & 8,7 & 19,6 & 8,4 \\
\hline \multicolumn{7}{|c|}{ Tipo de hospital (número de camas) } \\
\hline Menos de 100 camas & 1.421 & 20,5 & 50,1 & 8,6 & 26,8 & 8,3 \\
\hline Entre 100 y 200 camas & 1.390 & 20,1 & 48,6 & 9 & 15,8 & 8,9 \\
\hline Más de 600 camas & 4.111 & 59,4 & 48,3 & 8,6 & 26,2 & 8,5 \\
\hline \multicolumn{7}{|l|}{ Características del hospital } \\
\hline Comarcal & 2.510 & 36,3 & 49,9 & 8,8 & 20,7 & 8,6 \\
\hline Terciario & 4.111 & 59,4 & 48,3 & 8,6 & 26,2 & 8,4 \\
\hline Monográfico & 301 & 4,3 & 44,6 & 8,5 & 26,9 & 8,2 \\
\hline $\mathrm{n}=6.922$ & & & $48,7(47,6-49,9)^{*}$ & $8,7(8,68-8,76)^{*}$ & $24,2(23,4-25,4)^{*}$ & $8,5(8,44-8,55)^{*}$ \\
\hline
\end{tabular}

* = Intervalo de confianza al 95\%

An. Sist. Sanit. Navar. 2009, Vol. 32, No 2, mayo-agosto 
Un 48,7\% de los pacientes recibió algún tipo de prescripción farmacológica (IC al 95\% 47,6-49,9). Un $24,2 \%$ de los pacientes recibió información sobre el régimen de vida (IC al 95\% 23,4-25,4). Los pacientes otorgaron una puntuación de 8,7 puntos a la calidad de la información sobre medicamentos (IC al $95 \%$ 8,68-8,76) y 8,5 puntos a la calidad de la información sobre el régimen de vida (IC al 95\% 8,44-8,55).

\section{Prescripción farmacológica}

Los resultados del análisis multinivel de los 2 modelos propuestos para esta variable están incluidos en la tabla 2.

Tabla 2. Análisis multinivel de la variable proporción de personas que han recibido prescripción farmacológica.

\begin{tabular}{|c|c|c|c|c|}
\hline \multirow{2}{*}{$\begin{array}{l}\text { Variable información medicamentos } \\
\text { Parte fija }\end{array}$} & \multicolumn{2}{|c|}{ Modelo vacío } & \multicolumn{2}{|c|}{ Modelo completo } \\
\hline & Coeficiente & Error estándar & Coeficiente & Error estándar \\
\hline Edad menos de 16 años & & & $-0,59$ & 0,17 \\
\hline Edad de 16 a 65 años & & & $-0,37$ & 0,06 \\
\hline Primera consulta & & & $-0,40$ & 0,06 \\
\hline \multirow[t]{2}{*}{ Servicio quirúrgico } & & & $-0,59$ & 0,25 \\
\hline & \multicolumn{2}{|c|}{ Varianza } & \multicolumn{2}{|c|}{ Varianza } \\
\hline Parte aleatoria & Valor & Error estándar & Valor & Error estándar \\
\hline Especialidad & 0,56 & 0,18 & 0,37 & 0,13 \\
\hline Unidad clínica & 0,12 & 0,04 & 0,11 & 0,03 \\
\hline Paciente & 3,29 & & 3,29 & \\
\hline Deviance & \multicolumn{2}{|c|}{$8.959,5$} & \multicolumn{2}{|c|}{$8.835,6$} \\
\hline $\begin{array}{l}\text { \% de variabilidad debida a la unidad } \\
\text { clínica }\end{array}$ & \multicolumn{2}{|c|}{$17,1 \%$} & \multicolumn{2}{|c|}{$12,6 \%$} \\
\hline $\begin{array}{l}\text { \% de variabilidad debida a la } \\
\text { especialidad }\end{array}$ & \multicolumn{2}{|c|}{$14,1 \%$} & \multicolumn{2}{|c|}{$9,7 \%$} \\
\hline $\begin{array}{l}\text { Variabilidad de las unidades clínicas } \\
\text { atribuible a la especialidad }\end{array}$ & \multicolumn{2}{|c|}{$82,1 \%$} & \multicolumn{2}{|c|}{$77,4 \%$} \\
\hline$\%$ de variabilidad debida al paciente & \multicolumn{2}{|c|}{$81,3 \%$} & \multicolumn{2}{|c|}{$87,4 \%$} \\
\hline
\end{tabular}

La parte fija del modelo comprende aquellas variables cuyo coeficiente ha resultado estadísticamente significativo. Para cada variable se especifica el valor del coeficiente junto con su error estándar.

Con respecto a la parte fija del modelo, las variables predictoras que han obtenido coeficientes estadísticamente significativos son la edad (menor prescripción si era menor de 65 años), el tipo de consulta (menor prescripción si era la primera consulta) y el tipo de servicio (menor prescripción si se trataba de un servicio quirúrgico).
La diferencia de desvianza entre el modelo vacío y el modelo completo ha sido de 123,9 puntos $(\mathrm{p}<0,001)$.

En el modelo completo, la unidad clínica ha explicado el $12,6 \%$ de la variabilidad sobre la prescripción farmacológica. El $77,4 \%$ de esa variabilidad se atribuye a la especialidad. Los pacientes han explicado el $87,4 \%$ de la variabilidad.

Los residuales del intercepto y sus intervalos de confianza se muestran en las figuras 2 y 3 . Las unidades clínicas y las especialidades situadas en el eje de abscisas 
están ordenadas de menor a mayor valor del intercepto.

Se observa que dos unidades clínicas han obtenido puntuaciones diferentes a la puntuación media (Fig. 2). Sin embargo, seis especialidades se sitúan por encima y cuatro por debajo de la media (Fig. 3).

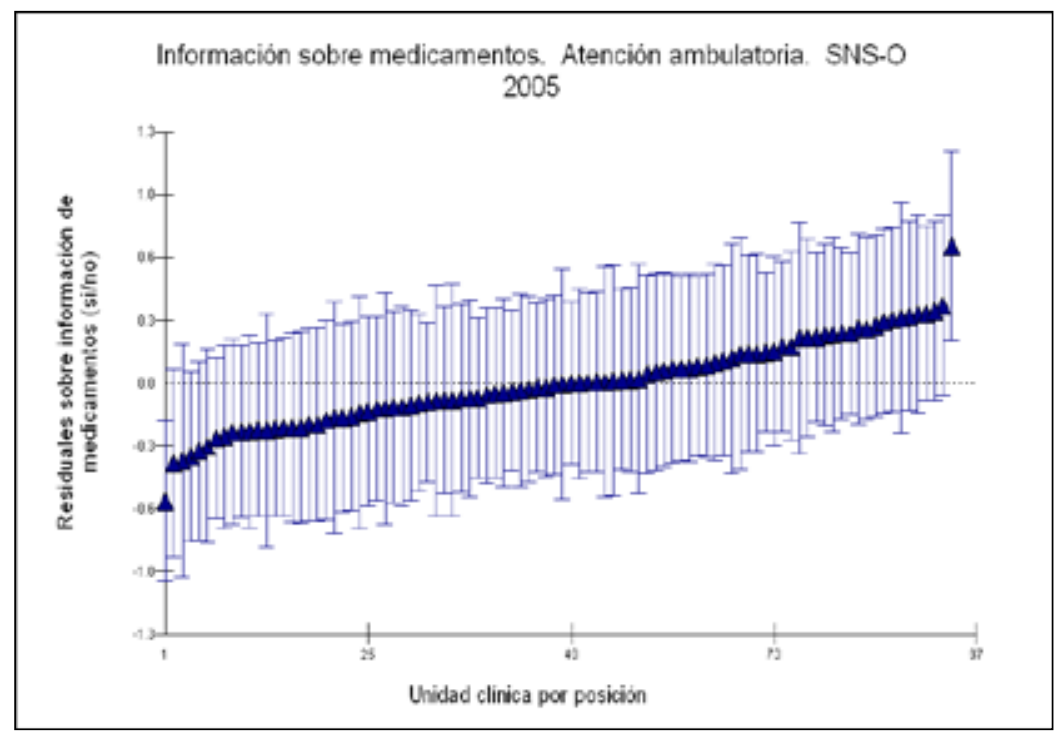

Figura 2. Residuales del intercepto de la variable proporción de personas que han recibido prescripción farmacológica. Modelo completo. Análisis por unidad clínica.

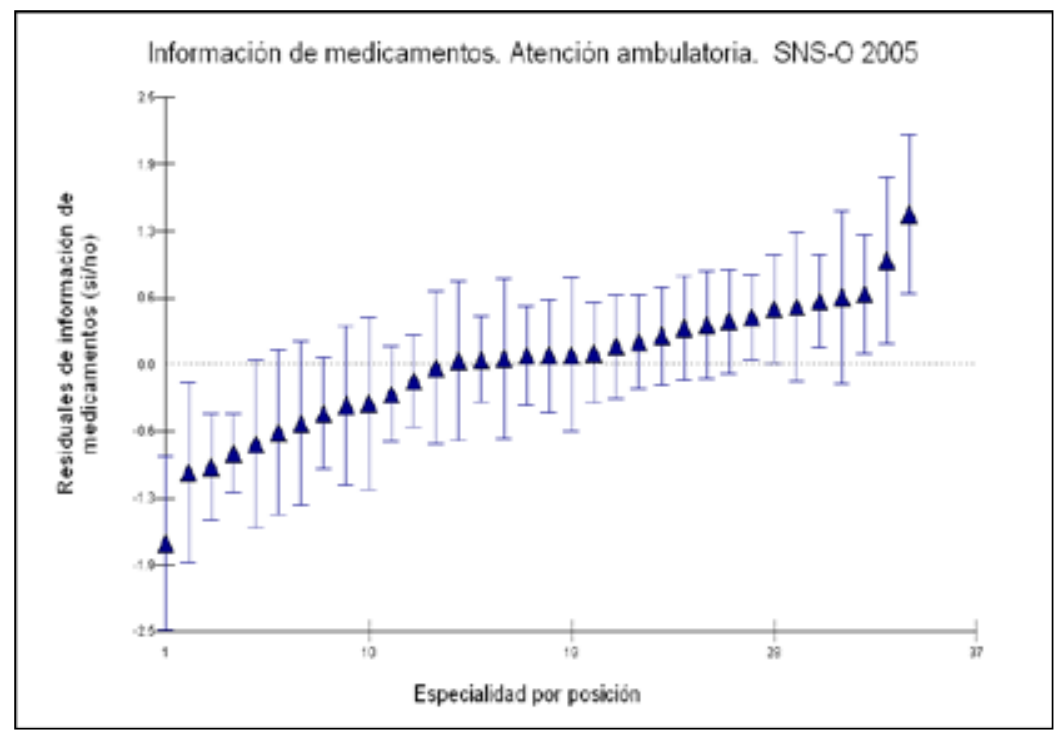

Figura 3. Residuales del intercepto de la variable proporción de personas han recibido prescripción farmacológica. Modelo completo. Análisis por especialidad. 


\section{Percepción de la calidad de la información sobre los medicamentos prescritos}

De acuerdo con lo observado en la parte fija del modelo completo que se muestra en la tabla 3 , solamente el coeficiente que hace referencia al tipo de hospital ha sido estadísticamente significativo (mejor percepción de la información sobre medicamentos si el centro está entre 100 y 200 camas).
La diferencia de desvianza entre el modelo vacío y el modelo completo es de 62,2 puntos $(\mathrm{p}<0,05)$.

Según se aprecia en la parte aleatoria del modelo completo, la unidad clínica ha explicado el $3 \%$ de la variabilidad de la percepción de la calidad de la información sobre los medicamentos prescritos y los pacientes el $97 \%$. La especialidad no ha conseguido explicar nada de esa variabilidad.

Tabla 3. Análisis multinivel de la variable calidad de la información recibida sobre la prescripción farmacológica.

\begin{tabular}{|c|c|c|c|c|}
\hline $\begin{array}{c}\text { Variable calidad información } \\
\text { medicamentos }\end{array}$ & \multicolumn{2}{|c|}{ Modelo vacío } & \multicolumn{2}{|c|}{ Modelo completo } \\
\hline Parte fija & Coeficiente & Error estándar & Coeficiente & Error estándar \\
\hline \multirow[t]{2}{*}{ Centro de 100 a 200 camas } & & & 0,36 & 0,10 \\
\hline & \multicolumn{2}{|c|}{ Varianza } & \multicolumn{2}{|c|}{ Varianza } \\
\hline Parte aleatoria & Valor & Error estándar & Valor & Error estándar \\
\hline Especialidad & 0,00 & 0,00 & 0,00 & 0,00 \\
\hline Unidad clínica & 0,06 & 0,01 & 0,04 & 0,01 \\
\hline Paciente & 1,19 & 0,03 & 1,18 & 0,03 \\
\hline Deviance & \multicolumn{2}{|c|}{$10.171,8$} & \multicolumn{2}{|c|}{$10.109,6$} \\
\hline $\begin{array}{l}\% \text { de variabilidad debida a la unidad } \\
\text { clínica }\end{array}$ & \multicolumn{2}{|c|}{$5,1 \%$} & \multicolumn{2}{|c|}{$3,0 \%$} \\
\hline $\begin{array}{l}\text { \% de variabilidad debida a la } \\
\text { especialidad }\end{array}$ & \multicolumn{2}{|c|}{$0,0 \%$} & \multicolumn{2}{|c|}{$0,0 \%$} \\
\hline $\begin{array}{l}\text { Variabilidad de las unidades clínicas } \\
\text { atribuible a la especialidad }\end{array}$ & \multicolumn{2}{|c|}{$0,0 \%$} & \multicolumn{2}{|c|}{$0,0 \%$} \\
\hline$\%$ de variabilidad debida al paciente & \multicolumn{2}{|c|}{$94,9 \%$} & \multicolumn{2}{|c|}{$97 \%$} \\
\hline
\end{tabular}

En la figura 4, que refleja los residuales del intercepto para cada unidad clínica, se detectan diferencias estadísticamente significativas entre unidades clínicas (6 unidades clínicas tienen puntuaciones inferiores a la media general).

\section{Información sobre el régimen de vida}

En la tabla 4 se muestran los resultados del análisis multinivel de los 2 modelos para la variable dependiente "información sobre el régimen de vida".
Las variables que han obtenido coeficientes estadísticamente significativos son: el género (menos información si era mujer), el tipo de consulta (menos información si era la primera consulta), el tipo de servicio (menos información si era un servicio quirúrgico), y el tipo de hospital (menos información si disponía entre 100 y 200 camas).

El modelo completo ha ajustado mejor que el modelo vacío ya que ha disminuido el valor de la desvianza en 109,1 puntos, siendo esta diferencia estadísticamente significativa $(\mathrm{p}<0,001)$. 


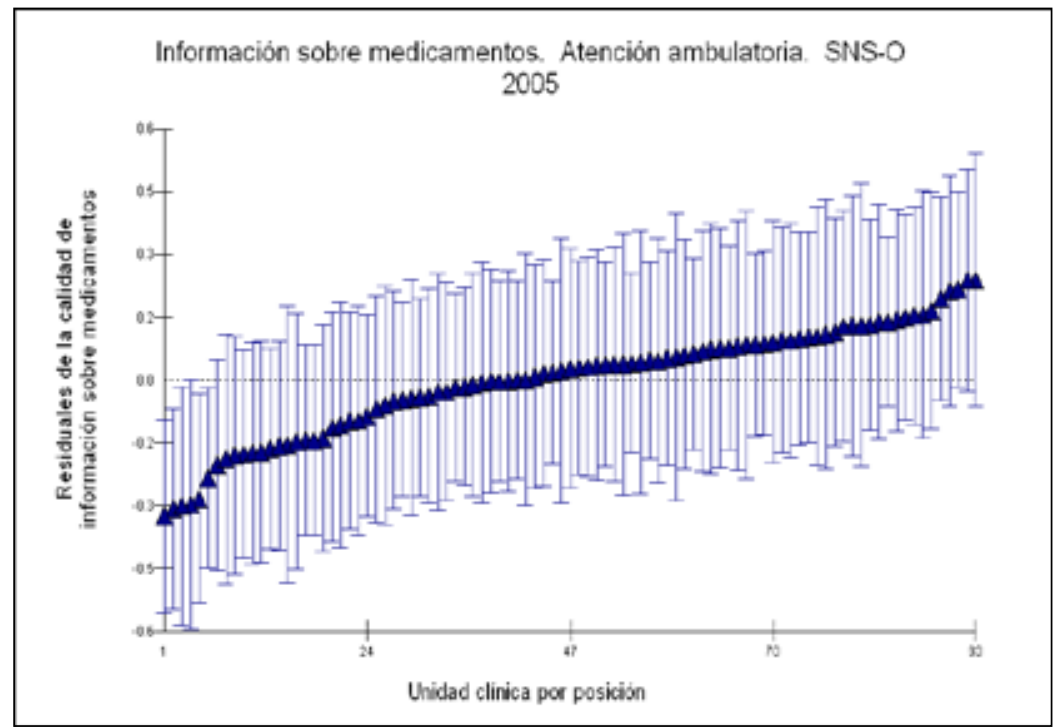

Figura 4. Residuales del intercepto de la variable calidad de la información sobre la prescripción farmacológica. Modelo completo. Análisis por unidad clínica.

Tabla 4. Análisis multinivel de la variable proporción de personas que habían recibido información sobre el régimen de vida.

\begin{tabular}{|c|c|c|c|c|}
\hline \multirow{2}{*}{$\begin{array}{l}\text { Variable información régimen de vida } \\
\text { Parte fija }\end{array}$} & \multicolumn{2}{|c|}{ Modelo vacío } & \multicolumn{2}{|c|}{ Modelo completo } \\
\hline & Coeficiente & Error estándar & Coeficiente & Error estándar \\
\hline Mujer & & & $-0,19$ & 0,06 \\
\hline Primera consulta & & & $-0,40$ & 0,05 \\
\hline Servicio quirúrgico & & & $-0,43$ & 0,17 \\
\hline \multirow[t]{2}{*}{ Centro de 100 a 200 camas } & & & $-0,77$ & 0,16 \\
\hline & \multicolumn{2}{|c|}{ Varianza } & \multicolumn{2}{|c|}{ Varianza } \\
\hline Parte aleatoria & Valor & Error estándar & Valor & Error estándar \\
\hline Especialidad & 0,12 & 0,07 & 0,12 & 0,05 \\
\hline Unidad clínica & 0,25 & 0,07 & 0,10 & 0,04 \\
\hline Paciente & 3,29 & & 3,29 & \\
\hline Deviance & \multicolumn{2}{|c|}{$7.371,9$} & \multicolumn{2}{|c|}{$7.262,8$} \\
\hline $\begin{array}{l}\text { \% de variabilidad debida a la unidad } \\
\text { clínica }\end{array}$ & \multicolumn{2}{|c|}{$10,0 \%$} & \multicolumn{2}{|c|}{$6,1 \%$} \\
\hline $\begin{array}{l}\text { \% de variabilidad debida a la } \\
\text { especialidad }\end{array}$ & \multicolumn{2}{|c|}{$3,2 \%$} & \multicolumn{2}{|c|}{$3,4 \%$} \\
\hline $\begin{array}{l}\text { Variabilidad de las unidades clínicas } \\
\text { atribuible a la especialidad }\end{array}$ & \multicolumn{2}{|c|}{$32,1 \%$} & \multicolumn{2}{|c|}{$55,0 \%$} \\
\hline \% de variabilidad debida al paciente & \multicolumn{2}{|c|}{$90 \%$} & \multicolumn{2}{|c|}{$93,9 \%$} \\
\hline
\end{tabular}


Tal y como se indica en el modelo completo, la unidad clínica ha explicado el 6,1\% de la variabilidad de la "información sobre el régimen de vida". El 55\% de esa variabilidad se atribuye a la especialidad. Los pacientes explicaron el $93,9 \%$ de la variabilidad.

En las figuras 5 y 6 se muestran los residuales del intercepto junto con sus intervalos de confianza para cada unidad clínica y especialidad, respectivamente. Como se puede observar en el la figura 5 , solamente una unidad clínica ha obtenido resultados significativamente inferiores a la media general. Sin embargo, en la figura 6 se observa que varias especialidades han obtenido diferencias significativas (4 especialidades se sitúan por encima y 3 por debajo de la media).

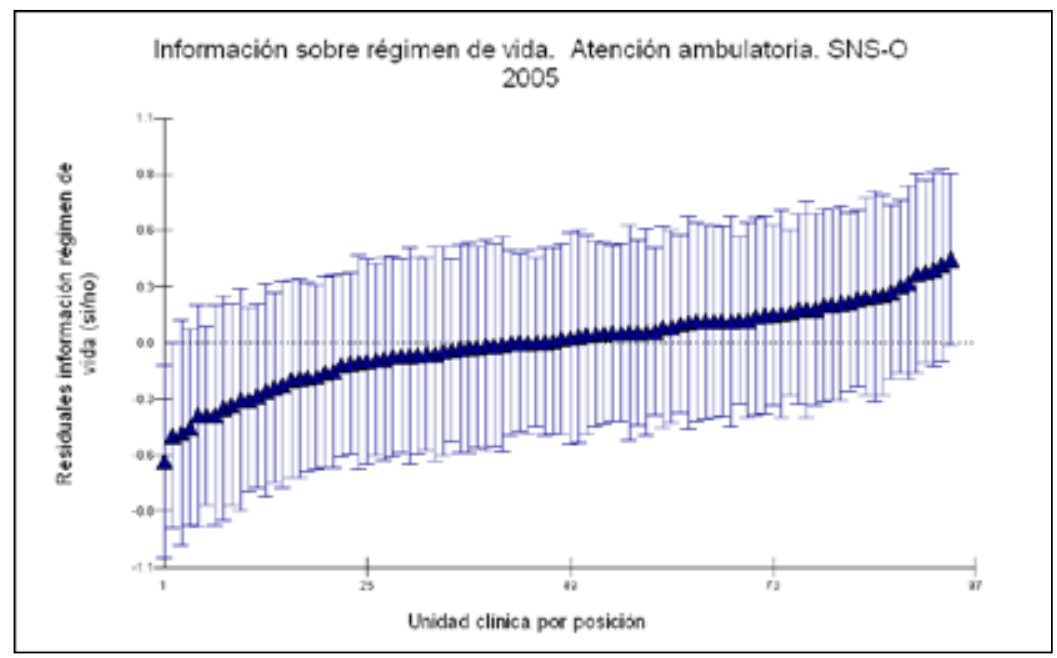

Figura 5. Residuales del intercepto de la variable proporción de personas que han recibido información sobre régimen de vida. Modelo completo. Análisis por unidad clínica.

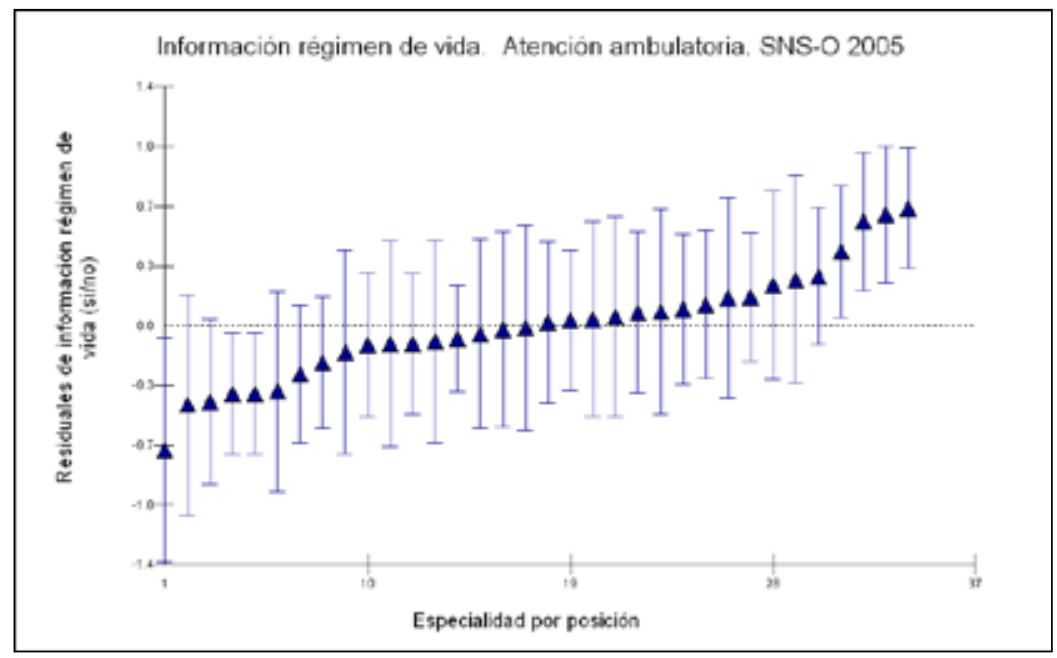

Figura 6. Residuales del intercepto de la variable proporción de personas que han recibido información sobre régimen de vida. Modelo completo. Análisis por especialidad. 
Percepción de la calidad de la información sobre régimen de vida

Los resultados del análisis multinivel de los 2 modelos propuestos para la variable calidad de la información sobre régimen de vida están especificados en la tabla 5 .

Tabla 5. Análisis multinivel de la variable calidad de la información recibida sobre el régimen de vida.

\begin{tabular}{|c|c|c|c|c|}
\hline $\begin{array}{l}\text { Variable calidad información } \\
\text { régimen de vida }\end{array}$ & \multicolumn{2}{|c|}{ Modelo vacío } & \multicolumn{2}{|c|}{ Modelo completo } \\
\hline Parte fija & Coeficiente & Error estándar & Coeficiente & Error estándar \\
\hline Edad de 16 a 65 años & & & 0,34 & 0,18 \\
\hline Más de 65 años & & & 0,40 & 0,18 \\
\hline \multirow[t]{2}{*}{ Entre 100 y 200 camas } & & & 0,63 & 0,12 \\
\hline & \multicolumn{2}{|c|}{ Varianza } & \multicolumn{2}{|c|}{ Varianza } \\
\hline Parte aleatoria & Valor & Error estándar & Valor & Error estándar \\
\hline Especialidad & 0,00 & 0,00 & 0,00 & 0,00 \\
\hline Unidad clínica & 0,05 & 0,02 & 0,01 & 0,01 \\
\hline Paciente & 1,21 & 0,04 & 1,21 & 0,04 \\
\hline Deviance & \multicolumn{2}{|c|}{$5.120,5$} & \multicolumn{2}{|c|}{$5.087,2$} \\
\hline $\begin{array}{l}\% \text { de variabilidad debida a la unidad } \\
\text { clínica }\end{array}$ & \multicolumn{2}{|c|}{$4,2 \%$} & \multicolumn{2}{|c|}{$0,7 \%$} \\
\hline $\begin{array}{l}\% \text { de variabilidad debida a la } \\
\text { especialidad }\end{array}$ & \multicolumn{2}{|c|}{$0,0 \%$} & \multicolumn{2}{|c|}{$0,0 \%$} \\
\hline $\begin{array}{l}\text { Variabilidad de las unidades clínicas } \\
\text { atribuible a la especialidad }\end{array}$ & \multicolumn{2}{|c|}{$0,0 \%$} & \multicolumn{2}{|c|}{$0,0 \%$} \\
\hline$\%$ de variabilidad debida al paciente & \multicolumn{2}{|c|}{95,8} & \multicolumn{2}{|c|}{99,3} \\
\hline
\end{tabular}

Con respecto a la parte fija del modelo, las variables predictoras que han obtenido coeficientes estadísticamente significativos son: la edad (mejor percepción si el paciente era mayor de 16 años) y el tipo de hospital (mejor percepción de la información sobre régimen de vida si tiene entre 100 y 200 camas).

La diferencia de desvianza entre el modelo vacío y el modelo completo ha sido de 33,3 puntos $(\mathrm{p}<0,05)$.

Tal y como se observa en la parte aleatoria del modelo completo, la unidad clínica ha explicado el $0,7 \%$ de la variabilidad de la percepción de la calidad de la información sobre el régimen de vida, y los pacientes el 99,3\%. La especialidad no ha conseguido explicar nada de esa variabilidad.

La figura 7 muestra los residuales del intercepto para cada unidad clínica. De acuerdo con lo allí observado, no se aprecian diferencias estadísticamente significativas entre unidades clínicas con respecto a la calidad de la información sobre el régimen de vida ofrecida a los pacientes. 


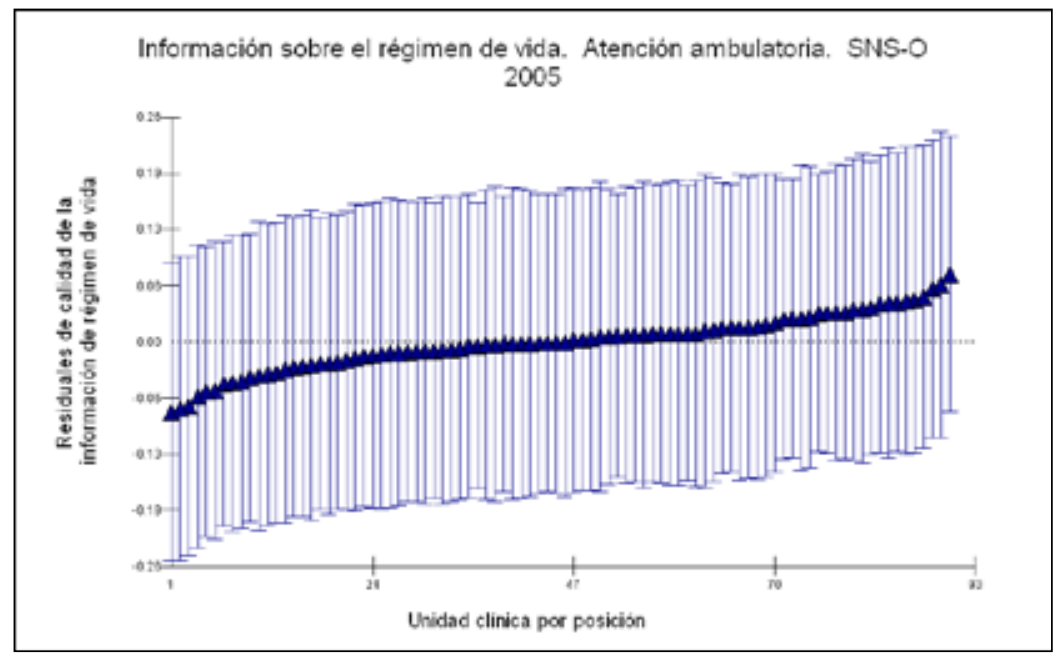

Figura 7. Residuales del intercepto de la variable calidad de la información sobre régimen de vida. Modelo completo. Análisis por unidad clínica.

\section{DISCUSIÓN}

El análisis multinivel se ha utilizado debido a que los pacientes atendidos en una misma unidad clínica o en una misma especialidad pueden compartir aspectos comunes, no pudiéndose garantizar la independencia de las observaciones. De igual forma, el comportamiento de los profesionales (información, empatía, etc.) podría diferir de unas unidades clínicas o especialidades médicas a otras ${ }^{20}$. El análisis multinivel tiene en cuenta la posibilidad de que los sucesos no sean independientes calculando de forma apropiada el error estándar cuando la estructura de los datos es jerárquica o anidada ${ }^{19,21}$. Además, esta técnica es especialmente recomendable cuando se introducen en el modelo variables cuyo valor es el mismo en cada grupo objeto de estudio (variables contextuales) ${ }^{18,21,22}$. El análisis multinivel requiere menor tamaño muestral dentro de cada grupo $^{18}$, posibilita ajustar de manera eficiente los diferentes tamaños entre los grupos ${ }^{18}$, permite realizar múltiples comparaciones ${ }^{23}$ -ya que tanto el estimador puntual como los intervalos de cada grupo se derivan de todos los datos y no solamente de los datos de un grupo concreto- y proporciona estimaciones más conservadoras ${ }^{19,23}$. Todos estos aspectos resultan especialmente importantes cuando el objetivo del análisis es realizar comparaciones entre unidades.

En este estudio la especialidad se ha utilizado como tercer nivel del modelo jerárquico. Las razones que condujeron a esta decisión fueron, por una parte, el hecho de que los pacientes atendidos por una misma especialidad pudieran compartir características comunes, y por otra, el que en ocasiones el comportamiento de los profesionales podría deberse más a las características específicas de la especialidad (en función de la formación recibida, tipo de pacientes que atiende ${ }^{24}$, cultura $^{24,25}$ ) que a las de una unidad clínica concreta.

Aunque el análisis multinivel ha permitido detectar unidades por encima y por debajo de los valores medios, la mayor parte de las diferencias obtenidas, al analizar las puntuaciones de la calidad de la información, podrían ser atribuidas a diferencias entre pacientes más que a diferencias entre unidades clínicas.

En los estudios revisados ${ }^{26-31}$ se han encontrado resultados similares a los obtenidos en este trabajo, oscilando la variabili- 
dad asignada a las unidades clínicas entre el $1 \%$ y el $10 \%$.

Las variaciones encontradas pueden deberse a diferentes causas: la primera haría referencia al tipo de instrumento utilizado (cuestionario para evaluar la calidad percibida); la segunda, a una considerable homogeneidad en la actuación de las diferentes unidades; y la tercera puede deberse a la gran variabilidad de comportamiento de los profesionales que pertenecen a las mismas unidades clínicas, lo que provocaría una regresión a la media en cada unidad.

La mayor parte de la variabilidad está en el nivel del paciente ya que, a diferencia de otros ámbitos como el educativo, los pacientes de las unidades clínicas no son reclutados de una unidad social concreta, y no suelen tener las mismas características socio-demográficas. Además los pacientes no interaccionan los unos con los otros lo suficiente como para que las evaluaciones y reacciones de los unos influyan en los otros. Los pacientes con patologías similares suelen ser tratados por las mismas unidades clínicas, pero no parece que la forma de interaccionar con los pacientes difiera de una unidad a otra ${ }^{19}$.

El análisis por especialidad nos ha indicado que ésta no explica la variabilidad de la calidad de la información recibida. Sin embargo, la especialidad tiene cierta importancia en la variabilidad de la prescripción farmacológica y en la de las recomendaciones sobre el régimen de vida. Es decir, unas especialidades prescriben más medicamentos o emiten más recomendaciones sobre el régimen de vida que otras. Sin embargo, la calidad de la información recibida por los pacientes que han recibido tratamiento farmacológico o recomendaciones sobre el régimen de vida no difiere entre especialidades.

Se ha comprobado que aquellas especialidades que "a priori" precisarían emitir más recomendaciones sobre el régimen de vida son las que más frecuentemente lo hacen (cardiología, endocrinología, etc.) frente a otras que lo precisarían menos (ORL, oftalmología). Una situación similar se da para la prescripción farmacológica ${ }^{24-32}$.
En cuanto a las variables explicativas, parecería lógico que los pacientes menores de 65 años requiriesen menos prescripción farmacológica. También podría ser posible que en las consultas sucesivas se disponga de una mayor precisión diagnóstica y que ello conduzca a que en ellas se faciliten al paciente más consejos sobre su régimen de vida y se lleve a cabo una mayor prescripción de fármacos. La confirmación de estas hipótesis requeriría la realización de un análisis de causalidad fuera del alcance del presente estudio.

Los pacientes han otorgado buenas puntuaciones tanto a la información sobre los medicamentos (8,7 puntos) como a la información sobre el régimen de vida $(8,5$ puntos). No obstante, resulta difícil realizar comparaciones con otros estudios debido a que las escalas y la formulación de las preguntas son diferentes. Según una encuesta de satisfacción de pacientes realizada en Inglaterra en el año $2005^{33}$, a un $28 \%$ de los pacientes se les prescribió medicación nueva durante la consulta externa. El $82 \%$ de estos pacientes percibió que la información recibida fue completa mientras que para el 13\% la información fue parcial.

Una de las posibles limitaciones del estudio es el número y tipo de variables utilizadas en el modelo. El modelo podría enriquecerse con la inclusión de un mayor número de variables que describieran con mayor detalle las características tanto de los pacientes como las de las unidades clínicas objeto de estudio.

La utilización de cuestionarios específicos que midiesen de forma más amplia la percepción de los pacientes sobre la medicación prescrita ${ }^{34,35}$ y sobre el régimen de vida proporcionaría una información más detallada que enriquecería el análisis.

Hay varias investigaciones que tratan de determinar cuáles son los métodos más adecuados para realizar comparaciones entre proveedores de servicios sanita$\operatorname{rios}^{36-43}$. Dado que las puntuaciones obtenidas por las diferentes unidades clínicas son muy similares ${ }^{44}$, la utilización de tablas de clasificación (league tables) no sería procedente $\mathrm{e}^{43-46}$. Sin embargo, el modelo de 
análisis multinivel resulta de gran interés para identificar las unidades clínicas con resultados excelentes y aquellas que requerirían acciones de mejora ${ }^{38}$. El uso del análisis multinivel permite la identificación de estas unidades clínicas con menos probabilidades de equivocación que si se emplean otros métodos ${ }^{17}$. Por lo tanto, este tipo de análisis es especialmente útil para llevar a cabo comparaciones entre unidades clínicas ${ }^{20-23}$.

La identificación de las unidades clínicas excelentes y de aquéllas con áreas de mejora es especialmente relevante en los casos en los que la organización establezca sistemas de recompensa (incentivos o penalizaciones) basados en la percepción de los pacientes, implante planes de mejora específicos para unidades clínicas en función de dichos resultados, establezca estrategias de benchmarking corporativo, o proporcione esta información a los pacientes quienes podrían incorporarla como un elemento más a la hora de elegir las unidades en las que desean ser atendidos.

Del conjunto del estudio, además de la alta calidad percibida por el paciente en la información recibida en relación a la prescripción medicamentosa y al estilo de vida recomendado, se puede deducir la homogeneidad en el comportamiento de las distintas unidades clínicas y especialidades.

\section{BIBLIOGRAFÍA}

1. HaLl J, DoRnan MC. Meta-analysis of satisfaction with medical care: description of research domain and analysis of overall satisfaction levels. Soc Sci Med 1988; 27: 637-644.

2. Garratt AM, Bjaertnes OA, Krogstad U, GulBRANDSEN P. The out patient experiences questionnaire (OPEQ): data quality, reliability, and validity in patients attending 52 Norwegian hospitals. Qual Saf Health Care 2005; 14 : 433-437.

3. Davies E, Cleary PD. Hearing the patient's voice? Factors affecting the use of patient survey data in quality improvement. Qual Saf Health Care 2005; 14: 428-432.

4. Petrella RJ, Lattanzio CN. Does counselling help patients get active? Can Fam Physician 2002; 48: 72-80.
5. CARR-HILL RA. The measurement of patient satisfaction. J Public Health 1992; 14: 236-249.

6. Williams S, Winman J, Dale J. Doctor-patient communication and patient satisfaction: a review. Family Practice 1988; 15: 480-492.

7. Barzilai DA, Goodwin MA, Zyszanski SJ, Stange KC. Does health habit counselling affect patient satisfaction? Prev Med 2001; 33: 595599.

8. Ziegler DK, Mosier MC, Buenaver M, OKuyemi $\mathrm{K}$. How much information about adverse effects of medication do patients want from physicians? Arch Intern Med 2001; 161: 706713.

9. Williams B. Patient satisfaction: A valid concept? Soc Sci Med 1994; 38: 509-516.

10. Crow R, Gage H, Hampson S, Hart J, Kimber A, Storey L et al. The measurement of satisfaction with healthcare: implications for practice from a systematic review of the literature. Health Technol Assess 2002; 6: 1-244.

11. Hendriks AAJ, Oort FJ, VRieling MR, Smets MA. Reliability and validity of the satisfaction with hospital care questionnaire. International Journal for Quality in Health Care 2002; 14: 471-482.

12. SitziA J. How valid and reliable are patient satisfaction data? An analysis of 195 studies. Int J Qual Health Care 1999; 11: 319-328.

13. WoOdside AG, Frey LL, DALY, RT. Linking service quality, customer satisfaction and behavioural intention. J Health Care Mark 1989; 9: 5-17.

14. Rasbash J, Browne W, Goldstein H, Yang M. Plewis II, Healy $M$ et al. A users guide to MLwiN. London: Institute of Education, 2000.

15. Hox JP. Multilevel analysis: techniques and applications. Manwah, NJ: Lawrence Erlbaum, 2002.

16. SNiJders T, Bosker RJ. Multilevel analysis. An introduction to basic and advanced multilevel modelling. LONDON: Sage publications, 1999.

17. Hox JJ, MAAs CJM. Multilevel analysis. Encyclopedia Social Measurement 2005; 2: 785793.

18. RASBAH J. Module 4: Multilevel structures and classifications. Centre for multilevel modelling. Bristol, 2008.

19. Hofoss D, Veenstra M, Krgogstad U. Multilevel analysis in health care research: a tutorial. Ann Ist Super Sanita 2003; 39: 213-222.

20. Kralewsky J, Dowd BE, Kaissi A, Curoe A, RocKWOOD T. Measuring the culture of medical 
group practices. Health care Manage Rev 2005; 30: 184-193.

21. Diez-Roux AV. Multilevel analysis in public health research. Annu Rev Public Health 2000; 21: 171-192.

22. Christiansen CL, Morris CN. Improving the statistical approach to health care provider profiling. Ann Intern Med 1997; 127: 764-768.

23. Tan A, Freeman JL, Freeman DH. Evaluating health care performance: strengths and limitations of multilevel analysis. Biom J 2007; 49: 707-718.

24. Yusuff KB, TAyo F. Does a physician's specialty influence the recording of medication history in patient's case notes? Br J Clin Pharmacol 2008; 66: 308-312.

25. Curoe A, Kralewski J, Kaissi A. Assessing the cultures of medical group practices. J Am Board Fam Pract 2003; 16: 394-398.

26. Sixma H, Spreeuwenberg PM. Patient satisfaction with the general practitioner: a two level analysis. Medical Care 1998; 36: 212-229.

27. SJetne IS, VeEnstra M. The effect of hospital size and teaching status on patient experiences with hospital care. Medical Care 2007; 45: 252-258.

28. Veenstra M, Hofoss D. Patient experiences with information in a hospital setting. Medical Care 2002; 41: 490-499.

29. Buorngaard JH, Ruud T, Garratt A, Hatling T. Patients' experiences and clinicians' ratings for the quality of outpatient teams in psychiatric care units in Norway. Psychiatr Serv 2007; 58: 1102-1107.

30. Aiello A, Garman A, Morris SB. Patient satisfaction with nursing care: a multilevel analysis. Q Manage Health Care 2003; 12: 187-190.

31. Swinkels ICS, Wimmers RH, Groenewegen PP, Van Den Bosch WJH, Dekker J, Van Den Ende CHM. What factors explain the number of physical therapy treatment sessions in patients referred with low back pain; a multilevel analysis. BMC Health Serv Res 2005, 5: 74.

32. Shah BR, Hus JE, Laupacis A, Ainman B, Booth GL. Use of vascular risk-modifying medications for diabetic patients differs between physician specialties. Diabetic Medicine 2008; 23: 1117-1123.

33. Commission for healthcare audit and inspection. Patient survey programme 2004/2005. Outpatient department: key findings. 2005. [Consultado 15-01.2008]: Disponi- ble en: http://www.cqc.org.uk/_db/_documents/04014871.pdf

34. Horne R, Hankins M, Henkins R. The Satisfaction with Information about Medicines Scale (SIMS): a new measurement tool for audit and research. Qual Health Care 2001; 10: 135140.

35. BARBER N. Ensuring patients' satisfaction with information about their medicines. Qual Health Care 2001; 10: 131-132.

36. Ones HE, Ohlssen DI, Spiegelhalter DJ. Use of the false discovery rate when comparing multiple health care providers. J Clin Epidemiol 2008; 61: 232-240.

37. Hammmed MA, Deens JJ. In the context of performance monitoring, the caterpillar plot should be mothballed in favour of the funnel plot. Ann Thorac Surg 2008; 86: 348.

38. Landrum MB, Bronskill SE, Normand SLT. Analytical methods for constructing rosssectional profiles for health care providers. Health Serv Outcome Res Meth 2000; 1: 2347.

39. Ohlssen DI, Sharples LD, Spiegelhalter DJ. A hierarchical modelling framework for identifying unusual performance in health care providers. J R Stat Soc Ser A Stat Soc 2007; 170: 865-890.

40. ReEs M, Dineschandra J. Monitoring clinical performance: the role of software architecture. Health Care Manag Sci 2008; 8: 197-203.

41. SpiegelHalter DJ. Funnel plots for comparing institutional performance. Statist Med 2005, 24; 1185-1202.

42. FREEMAN T. Using performance indicators to improve health care quality in public sector: a review of the literature. Health Serv Manag Res 2002; 15: 126-137.

43. Rogers G, Smith DD. Reporting comparative results form hospital patient surveys. Int $\mathrm{J}$ Qual Health Care 1999; 11: 251-259.

44. Williams B, Coyle J, Healy D. The meaning of patient satisfaction: An explanation of high reported levels. Soc Sci Med 1998; 47: 13511359.

45. Goldsten H, Spiegelhalter DJ. League tables and their limitations: statistical issues in comparisons of institutional performance. $\mathrm{J}$ R Stat Soc Ser A Stat Soc 1996; 159: 385-409.

46. Bridgewater B, Keogh B. Surgical "league tables": ischemic heart disease. Heart 2008; 94 : 936-942. 
